\title{
NEW APPLICATIONS OF RADIOGUIDED SURGERY IN ONCOLOGY
}

\author{
Almir Galvão Vieira Bitencourt, ${ }^{\text {I }}$ Eduardo Nóbrega Pereira Lima, ${ }^{\text {II Paula }}$ \\ Nicole Vieira Pinto, ${ }^{\mathrm{I}}$ Eduardo Bruno Lobato Martins, ${ }^{\mathrm{I}}$ Rubens Chojniak ${ }^{\mathrm{I}}$
}

doi: $10.1590 / \mathrm{S} 1807-59322009000500005$

Bitencourt AGV, Lima ENP, Pinto PNV, Martins EBL, Chojniak R. New applications of radioguided surgery in oncology. Clinics. 2009;64(5):397-402.

OBJECTIVE: To report oncological cases (excluding those related to breast cancer) for which radioguided surgery has been used in combination with the Radioguided Occult Lesion Localization technique.

INTRODUCTION: Radioguided surgery enables a surgeon to identify lesions or tissues that have been preoperatively marked with radioactive substances. The Radioguided Occult Lesion Localization technique has been widely used to identify the sentinel lymph node and occult lesions in patients with breast cancer. However, few studies have reported the use of this technique for non-breast cancer pathologies.

METHODOLOGY: In all cases, injection of Technecium-99m sulfur colloid was performed, directly inside or near by the suspicious lesion, guided by ultrasound or computed tomography, up to 36 hours prior to the surgical procedure. Intraoperative lesion detection was carried out using a gamma-probe.

RESULTS: We report five oncology cases in which preoperative markings of the lesions were carried out using the Radioguided Occult Lesion Localization technique. The patients presented with the following: recurrence of renal cell carcinoma, cervical recurrence of papillary carcinoma of the thyroid, recurrence of retroperitoneal sarcoma, lesions of the popliteal fossa, and recurrence of rhabdomyosarcoma of a thigh. In each case, the lesions that were marked preoperatively were ultimately successfully excised.

CONCLUSIONS: Radioguided surgery has proven to be a safe and effective alternative for the management of oncology patients. The Radioguided Occult Lesion Localization technique can be useful in selected cases where suspect lesions may be difficult to identify intraoperatively, due to their dimensions or anatomical location. The procedure allows for more conservative excisions and reduces the surgery-related morbidity.

KEYWORDS: Radioguided surgery; Radioguided occult lesion localization; Gamma-detecting probe; Surgical Oncology; Technetium Tc 99m Sulfur Colloid.

\section{INTRODUCTION}

Radioguided surgery allows a surgeon to identify lesions or tissues that have been preoperatively marked with a radioactive substance. The intraoperative detection of these structures is performed using a gamma-probe, which leads to smaller and less traumatic excisions. At present, the main clinical application of this surgical technique in oncology is

\footnotetext{
${ }^{I}$ Department of Image, Hospital AC Camargo - São Paulo/SP, Brazil.

II Nuclear Medicine, Hospital AC Camargo - São Paulo/SP, Brazil.

Email: almirgvb@yahoo.com.br

Tel.: 55112189.5000

Received for publication on November 28, 2008

Accepted for publication on February 03, 2009
}

to identify the sentinel lymph node and occult lesions or to find lesions that are difficult to locate intraoperatively.

The identification of occult lesions for radioguided surgery can be carried out in two ways. The first approach consists of systemic administration of a radiocolloid associated with a carrier that exhibits tropism for a specific tumor or tissue, such as $99 \mathrm{mTc}$-sestamibi for the identification of parathyroid adenomas or hyperplasia, or 111 In-DTPA octreotide for the identification of neuroendrocrine tumors., ${ }^{2,3}$ Under the second approach, the lesion to be excised is marked by intratumoral injection of the radioisotope, which is guided by an imaging method. This technique, known as Radioguided Occult Lesion Localization (ROLL), was first described in 1998 for the 
preoperative localization of occult lesions of the breast. ${ }^{4}$

The ROLL technique has routinely been used in many specialized centers for the preoperative marking of occult breast lesions and has demonstrated similar effectiveness and various advantages over metallic wire localization. ${ }^{5,6}$ However, few studies have reported the use of this technique for other pathologies. ${ }^{7}$ The objective of this work is to report oncological cases not related to breast cancer in which radioguided surgery has been performed using the ROLL technique.

\section{MATERIALS AND METHODS}

In this study, we report five oncology cases not related to breast cancer in which preoperative marking of lesions was performed using the ROLL technique. The patients presented suspect lesions identified by imaging methods such as ultrasound (US), computed tomography (CT) or magnetic resonance imaging (MRI), and positron emission tomography/CT (PET-CT) with fluorodeoxyglucose (18F-FDG). Since these lesions are difficult to identify intraoperatively due to their dimensions or localization, radioguided surgery was adopted in all cases.

Injection of 0.1 to $0.2 \mathrm{ml}$ of Technetium $99 \mathrm{~m}$ sulfur colloid (Sn-99mTc) with an activity count of 0.5 to 1.0 $\mathrm{mCi}$ (18.5 to $37 \mathrm{MBq}$ ) was administered (guided by US or CT) directly into or next to the suspect lesions up to 36 hours prior to surgery. Immediately after injection of the radioactive material, single photon emission computed tomography/CT (SPECT-CT) imaging was performed to confirm the location and technical quality of the injection. The marked lesions were identified intraoperatively using a gamma-probe and were excised with safety margins. The radioactivity of the tissue was measured in vivo and after excision, as well as the radioactivity of the surgical bed, to confirm that the marked lesion had been fully excised.

\section{RESULTS}

\section{Case 1}

Patient ZDF, male, aged 50 years, was admitted with a history of right nephrectomy for renal cell carcinoma performed eight months previously. CT of the abdomen on follow-up identified a hypervascularized heterogeneous nodule in the right retroperitoneum, measuring $23 \times 18$ $\mathrm{mm}$, and two nodules in the lumbar wall, measuring 8 and $17 \mathrm{~mm}$ (Figure 1A). PET-CT confirmed the presence of all lesions on account of high concentrations of 18F-FDG. CT-guided percutaneous biopsies were performed and revealed suggestive infiltration by clear cell renal carcinoma. The patient was then submitted to excision of the lesions of the abdominal wall and of the retroperitoneal nodule using a gamma-probe to detect the lesions that had been preoperatively marked using CT-guided ROLL (Figure 1B). Surgery lasted for one hour and forty minutes, and discharge occurred after five days without complications. Histopathological analysis of the surgical specimens with immunohistochemistry confirmed the hypothesis of recurrence of clear cell renal carcinoma.

\section{Case 2}

NG, female, aged 39 years, was admitted for thyroidectomy and right neck dissection for multifocal classical papillary carcinoma with lymph node metastasis. During the follow-up, an increase of thyrogobulin was evidenced, and cervical US identified enlarged lymph nodes in the right jugular chain (Level IV), above the subclavian veins, measuring $14 \mathrm{~mm}$ at their smallest axis. The scintigraphy with iodine-131 did not show any alterations, but the PET-CT demonstrated an anomalous concentration of $18 \mathrm{~F}-\mathrm{FDG}$ in the enlarged right cervical lymph nodes

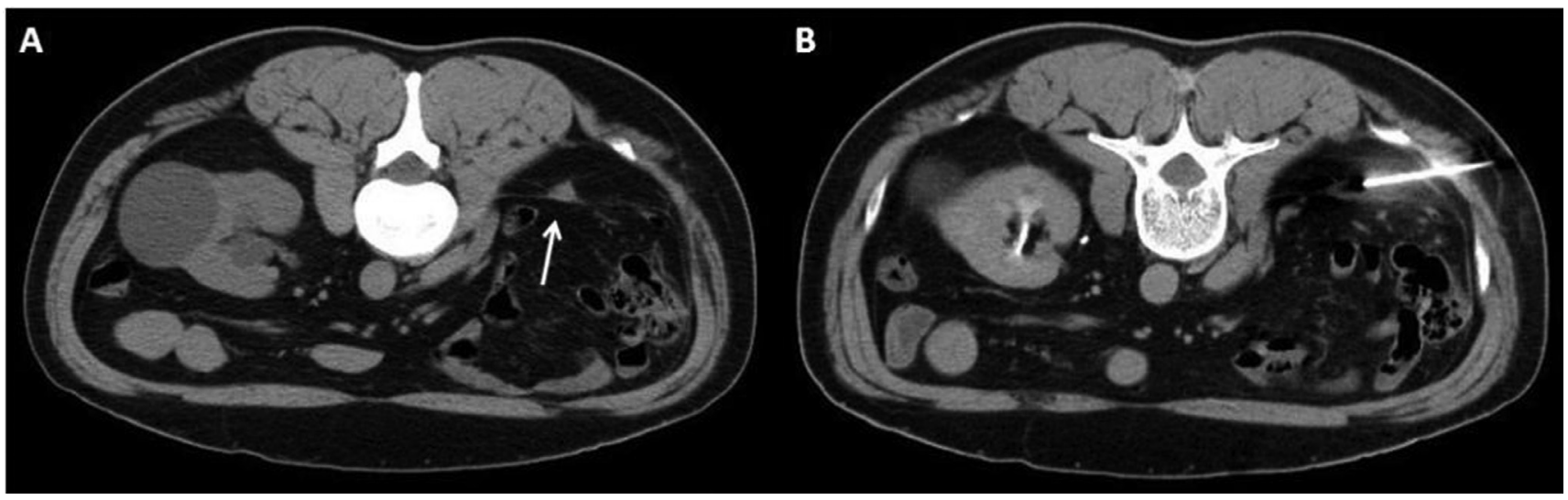

Figure 1 - A) Computed tomography of the abdomen without contrast IV injection in the ventral decubitus with nodular imaging in the right renal sinus (arrow). B) Moment of CT-guided injection of $\mathrm{Sn}-{ }^{99 \mathrm{~m}} \mathrm{Tc}$ into the lesion 


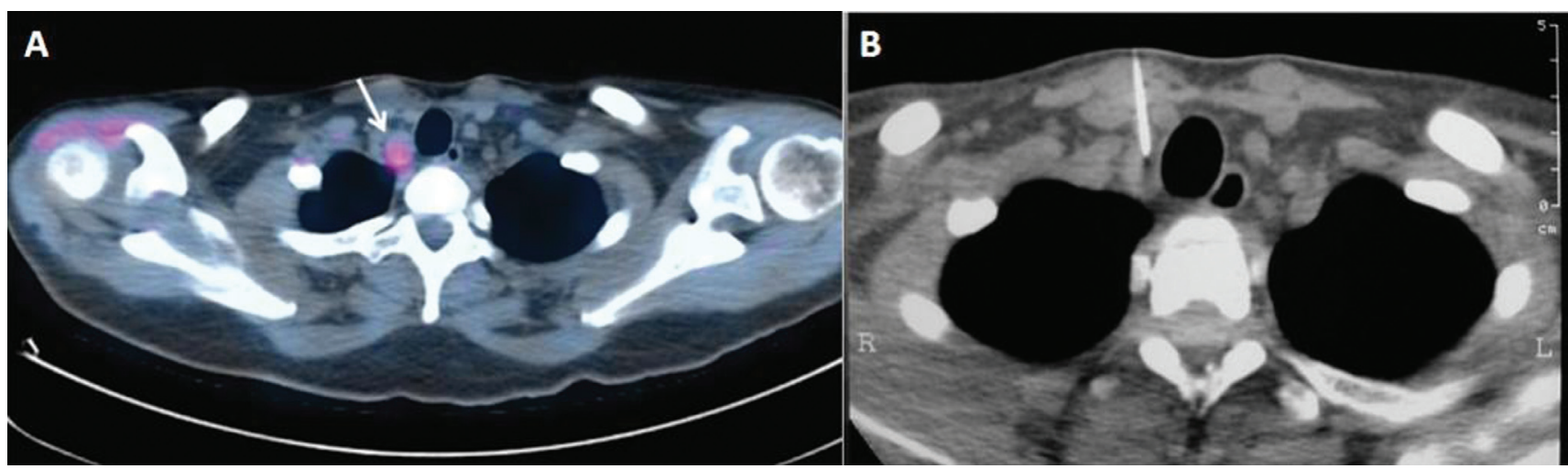

Figure 2 - A) PET-CT demonstrating an anomalous concentration of ${ }^{18} \mathrm{~F}-\mathrm{FDG}$ in the enlarged right cervical lymph node (maximum SUV: 1.65 ). B) Moment of CT-guided injection of Sn- ${ }^{99 m} \mathrm{Tc}$ into the lesion

(Figure 2A). This lesion was excised after preoperative localization through CT-guided intralesional injection of Sn-99mTc (Figure 2B). The surgery lasted two hours and forty minutes. No complications were recorded, and the patient was discharged on the first post-operative day. The histopathological analysis confirmed metastasis of papillary thyroid carcinoma on the cervical lymph node.

\section{Case 3}

HHFF, female, aged 63 years, was admitted with a history of a high-grade retroperitoneal pleomorphic sarcoma that had been treated two years previously with neoadjuvant chemotherapy, surgery (monoblock excision of the retroperitoneal tumor, psoas muscle, and a segment of the left femoral nerve), and intraoperative radiotherapy. Four months prior to admission, the patient had undergone videoassisted excision of a right lung metastasis. In the PET-CT control exam, areas of anomalous 18F-FDG concentrations were detected in the left iliac fossa, involving the femoral nerve stump and infiltrating the oblique muscle, next to the iliac, and in the left posterolateral abdominal wall, adhered to the end of the 12th rib, adjacent to the lower renal pole. CT-guided percutaneous biopsy of the lesion in the iliac fossa tested positive for sarcoma. CT-guided Sn-99mTc injection was administered in the lesions and was confirmed using SPECT-CT (Figure 3) prior to surgery. The patient underwent excision of both lesions, using a gamma-probe and intraoperative radiotherapy. The surgery lasted four hours and thirty-five minutes, and the patient was discharged on the seventh post-operative day without complications.

\section{Case 4}

LAAR, male, aged 40 years, without any oncological history, complained of pain in the distal portion of the thigh and edema of the right leg for one month. MRI identified two expansive solid formations in the vasculonervous axis of the right popliteal fossa, one higher up, measuring 31 x $25 \mathrm{~mm}$, and the other lower down, measuring $26 \times 19$ $\mathrm{mm}$. We decided that surgical treatment with preoperative marking of the lesions with ROLL should be performed. US-guided injection of Sn-99mTc was performed on the lower lesion and was confirmed by SPECT-CT (Figure

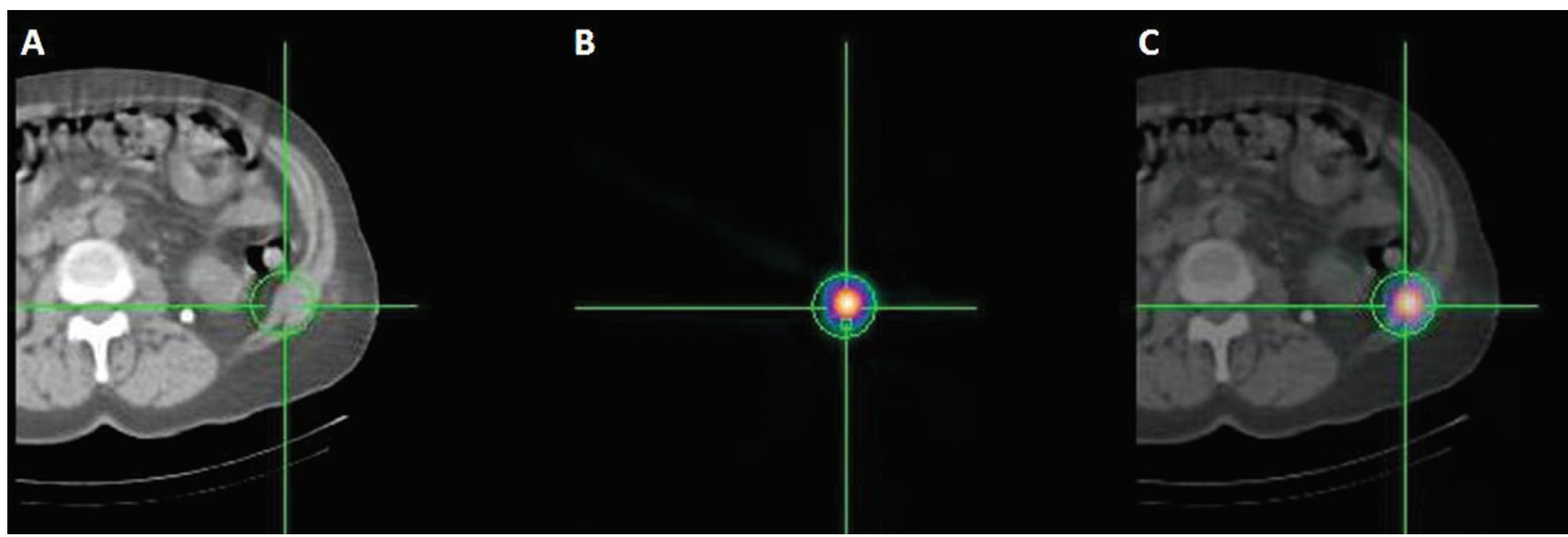

Figure 3 - SPECT-CT performed after Sn- ${ }^{99 \mathrm{~m}} \mathrm{Tc}$ injection into the lesion of the abdominal wall, left side. A) Anatomical image. B) Metabolic image. C) Fusion of the two methods 

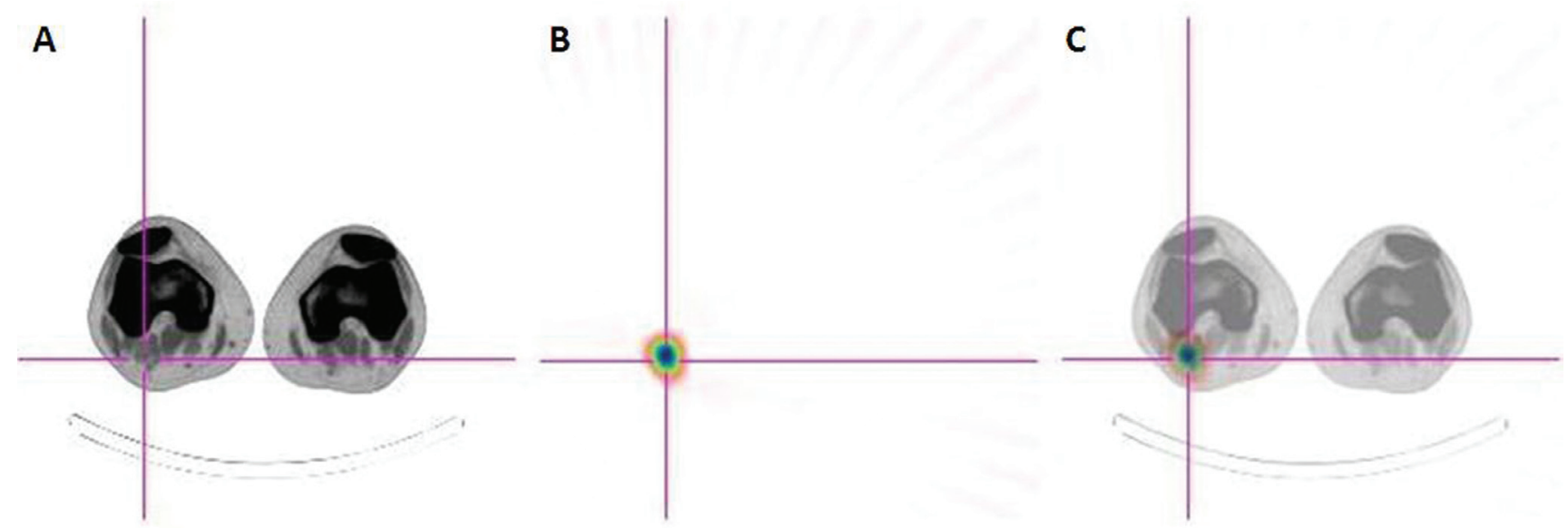

Figure 4 - SPECT-CT performed after Sn- ${ }^{99 m}$ Tc injection in the lesion of the right popliteal fossa. A) Anatomical image. B) Metabolic image. C) Fusion of the two methods

4). The upper lesion, located approximately $50 \mathrm{~mm}$ above the lower one, was not marked due to its proximity to the popliteal veins. The surgery lasted for two hours and fifteen minutes, and the length of the hospital stay was two days. There were no immediate post-operative complications. The histopathological analysis of both lesions revealed that these were schwannomas.

\section{Case 5}

SBCP, female, aged 16 years, with a history of tumors of the left thigh, submitted to extended excision one year ago, for which the histological diagnosis was embryonic rhabdomyosarcoma. Six months ago, the patient underwent marginal excision of recurrence in the topography of the left femoral vein. In the oncological follow-up, PET-CT was performed, evidencing an anomalous concentration of 18F-FDG in the topography of the subcicatricial nodule deep in the left thigh. The lesion was marked with USguided injection of Sn-99mTc and confirmed by SPECT-CT
(Figure 5). The patient subsequently underwent radioguided marginal excision. Surgery took about two hours and forty minutes without complications. The patient was discharged on the second post-operative day. The histopathological analysis confirmed undifferentiated round cell neoplasia infiltrating soft parts, compatible with a recurrence of rhabdomyosarcoma.

\section{DISCUSSION}

The ROLL technique used in these patients was effective in the intraoperative localization of the marked lesions, demonstrating that this is a feasible and promising technique for the surgical exploration of selected cases. This technique does not have any side effects, requires minimal radiation, and enables less invasive procedures with a lower level of post-operative complications. The approach reduces surgery time and shortens a patient's hospital stay. ${ }^{8}$

Technical precautions at the moment of administration of the radiocolloid and at the time of preoperative imaging

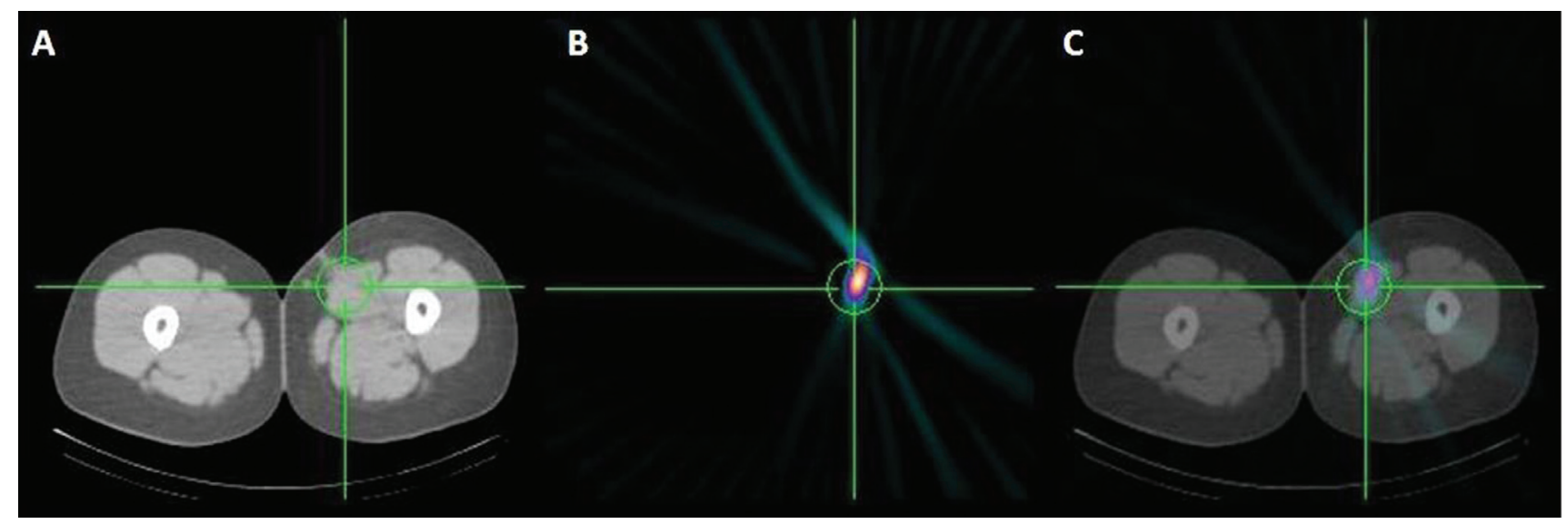

Figure 5 - SPECT-CT carried out after Sn- ${ }^{99 \mathrm{~m}} \mathrm{Tc}$ injection in the left abdominal lesion. A) Anatomical image. B) Metabolic image. C) Fusion of the two methods 
are essential for guaranteeing the intraoperative localization without complications. The preoperative image is important for confirming the technical quality of the injection, ruling out the possibility of leakage, contamination involving the skin, and residual activity in the path of the needle. This imaging should preferably be carried out with SPECT-CT, which enables simultaneous observation of the distribution of the radiocolloid and its precise anatomical location provided by a CT device coupled with a SPECT gamma-camera.

Despite being used successfully in cases of occult breast lesions, the ROLL technique has been rarely described in other oncological pathologies. Gulec et al. (2007) describe three non-habitual oncology cases, in which radioguided surgery was carried out after injection of an albumin macroaggregate following the US- or endoscopy-guided injection of ${ }^{99 \mathrm{~m}} \mathrm{Tc}$. In two of these cases, the surgical indication was for the diagnostic investigation of suspect enlarged lymph nodes, one axillary in a patient with a history of breast cancer, and the other mediastinal in a patient with a history of non-Hodgkin's lymphoma. In the third case, the surgical indication was to determine the extent of the surgical excision in a patient with colonic polyposis. ${ }^{7}$

In cases of cervical recurrence of well-differentiated thyroid cancer, some authors have demonstrated good results with radioguided surgery following systemic administration of iodine-131. ${ }^{910}$ However, in cases where the lesion does not concentrate iodo-131 in the scintigraphy, as in the second case presented here, this methodology is ineffective. Other authors have also reported a successful use of the ROLL technique in patients with recurrence of thyroid cancer in non-palpable cervical lymph nodes. ${ }^{11,12}$
In four of the five cases presented, the lesions were identified preoperatively by PET-CT. With the growing use of this method in the evaluation of oncological patients, some authors have described techniques for intraoperative localization of recurring or metastatic lesions identified by the PET-CT. ${ }^{13-16}$ Given that ${ }^{18} \mathrm{~F}$-FDG has a short halflife of around two hours, the systemic injection of this radiocolloid should be carried out immediately before surgery. Furthermore, as the photons liberated by the ${ }^{18} \mathrm{~F}$-FDG have $511 \mathrm{keV}$ of energy, compared with $140 \mathrm{keV}$ for photons liberated by ${ }^{99 \mathrm{~m}} \mathrm{Tc}$, it is necessary to use a specific high-energy gamma-probe. ${ }^{17}$ However, this methodology requires further study to prove its effectiveness. The ROLL technique therefore provides an interesting alternative for the intraoperative localization of these lesions.

Other recently described applications of the ROLL technique include localization of pulmonary nodules during thoracoscopy ${ }^{18,19}$ and identification of non-palpable colonic lesions during open or laparoscopic surgery following colonoscopy-guided radiocolloid injection. ${ }^{20}$

\section{CONCLUSIONS}

Radioguided surgery has proven to be a feasible and promising alternative for the management of oncological patients. The ROLL technique may be useful in selected cases where the suspect lesions are difficult to identify intraoperatively due to their dimensions or anatomical location, enabling more conservative excisions and reducing the morbidity related to surgery.

\section{REFERENCES}

1. Schneebaum S, Even-Sapir E, Cohen M, Shacham-Lehrman H, Gat A, Brazovsky E, et al. Clinical applications of gamma-detection probes radioguided surgery. Eur J Nucl Med. 1999;26:S26-35.

2. Mariani G, Gulec SA, Rubello D, Boni G, Puccini M, Pelizzo MR, et al. Preoperative localization and radioguided parathyroid surgery. J Nucl Med. 2003;44:1443-58.

3. Gulec SA, Baum R. Radio-guided surgery in neuroendocrine tumors. J Surg Oncol. 2007;96:309-15.

4. Zurrida S, Galimberti V, Monti S, Luini A. Radioguided localization of occult breast lesions. Breast. 1998;7:11-3.

5. Rampaul RS, Bagnall M, Burrell H, Pinder SE, Evans AJ, Macmillan RD. Randomized clinical trial comparing radioisotope occult lesion localization and wire-guided excision for biopsy of occult breast lesions. Br J Surg. 2004;91:1575-7.
6. Medina-Franco H, Abarca-Pérez L, García-Alvarez MN, Ulloa-Gómez JL, Romero-Trejo C, Sepúlveda-Méndez J. Radioguided occult lesion localization (ROLL) versus wire-guided lumpectomy for non-palpable breast lesions: a randomized prospective evaluation. J Surg Oncol. 2008;97:108-11.

7. Gulec SA, Hoenie E, Rheinheimer K. A multimodality localization technique for radio-guided surgery. World J Surg Oncol. 2007;5:43-7.

8. Thind CR, Desmond S, Harris O, Nadeem R, Chagla LS, Audisio RA. Radio-guided localization of clinically occult breast lesions (ROLL): a DGH experience. Clin Radiol. 2005;60:681-6.

9. Travagli JP, Cailleux AF, Ricard M, Baudin E, Caillou B, Parmentier $\mathrm{C}$, et al. Combination of radioiodine (131I) and probe-guided surgery for persistent or recurrent thyroid carcinoma. J Clin Endocrinol Metab. 1998;83:2675-80. 
10. Salvatori M, Rufini V, Reale F, Gajate AMS, Maussier ML, Revelli L, et al. Radio-guided Surgery for Lymph Node Recurrences of Differentiated Thyroid Cancer. World J Surg. 2003;27:770-5.

11. Tükenmez M, Erbil Y, Barbaros U, Dural C, Salmaslioglu A, Aksoy D, et al. Radio-guided nonpalpable metastatic lymph node localization in patients with recurrent thyroid cancer. J Surg Oncol. 2007;96:534-8.

12. Raschillà R, Manca G, Grosso M, Borsò E, Volterrani D, Dell'Anno $\mathrm{B}$, et al. A novel procedure for radio-guided surgery of non-palpable cervical recurrence of thyroid cancer . Annual Congress of the European Association of Nuclear Medicine 2008;TP24.

13. Gulec SA, Hoenie E, Hostetter R, Schwartzentruber D. PET probeguided surgery: applications and clinical protocols. World J Surg Oncol. 2007;5:65-71

14. Essner R, Daghighian F, Giuliano AE: Advances in FDG PET probes in surgical oncology. Cancer J. 2002;8:100-8.

15. Sarikaya I, Povoski SP, Al-Saif OH, Kocak E, Bloomston M, Marsh $\mathrm{S}$, et al. Combined use of preoperative 18F FDG-PET imaging and intraoperative gamma probe detection for accurate assessment of tumor recurrence in patients with colorectal cancer. World J Surg Oncol. 2007;5:80-8.
16. Piert M, Burian M, Meisetschläger G, Stein HJ, Ziegler S, Nährig J, et al. Positron detection for the intraoperative localisation of cancer deposits. Eur J Nucl Med Mol Imaging. 2007;34:1534-44.

17. Gulec SA. PET probe-guided surgery. J Surg Oncol. 2007;96:353-7.

18. Sortini D, Feo C, Maravegias K, Carcoforo P, Pozza E, Liboni A, et al. Intrathoracoscopic localization techniques. Review of literature. Surg Endosc. 2006;20:1341-7.

19. Daniel TM, Altes TA, Rehm PK, Williams MB, Jones DR, Stolin AV, et al. A novel technique for localization and excisional biopsy of small or ill-defined pulmonary lesions. Ann Thorac Surg. 2004;77:1756-62.

20. Rezzo R, Scopinaro G, Gambaro M, Michetti P, Anfossi G. Radioguided occult colonic lesion identification (ROCLI) during open and laparoscopic surgery. Tumori. 2002;88:S19-22. 